\section{Digital dental photography. Part 8: intra-oral set-ups}

\section{Ahmad'}
IN BRIEF
- Once an understanding of the basic set- ups is gained, intra-oral photography can be expedited in a few minutes.
- Cross-infection control during a photographic session is mandatory.
- As well as taking 'stock' dental images, one can concentrate on specific points of interest and analyse them later.
- Intra-oral photography is also an excellent method of communication with the patient, ceramist and specialist.

The majority of pictures taken in the dental surgery are intra-oral and this article looks at the practicalities involved in fullarch, quadrant occlusal, lingual (or palatal) and lateral views, as well as magnified images, oral mucosa, enamel texture, dentine strata and shade analysis for artificial restorations. Additionally, the issue of cross-infection control is considered.

The majority of pictures taken in the dental surgery are intra-oral, which includes the following:

1. Full arch - frontal and occlusal

2. Quadrant occlusal, lingual

(or palatal) and lateral

3. Magnification images for detailed analysis of teeth or soft tissues

4. Oral mucosa

5. Enamel texture, cracks and dentine strata

6. Translucency: incisal and interproximal

7. Shade analysis for artificial restorations

8. Posterior teeth.

\section{FUNDAMENTALS OF DIGITAL DENTAL PHOTOGRAPHY}

1. Digital dental photography: an overview

2. Purposes and uses

3. Principles of digital photography

4. Choosing a camera and accessories

5. Lighting

6. Camera settings

7. Extra-oral set-ups

8. Intra-oral set-ups

9. Post-image capture processing

10. Printing, publishing and presentations

General Dental Practitioner, The Ridgeway Dental Surgery, 173 The Ridgeway, North Harrow, Middlesex, HA2 7DF

Correspondence to: Irfan Ahmad

Email: iahmadbds@aol.com

www.IrfanAhmadTRDS.co.uk

\section{Refereed Paper}

Accepted 15 November 2008

DOI: $10.1038 /$ sj.bdj.2009.715

${ }^{\circledR}$ British Dental Journal 2009; 207: 151-157

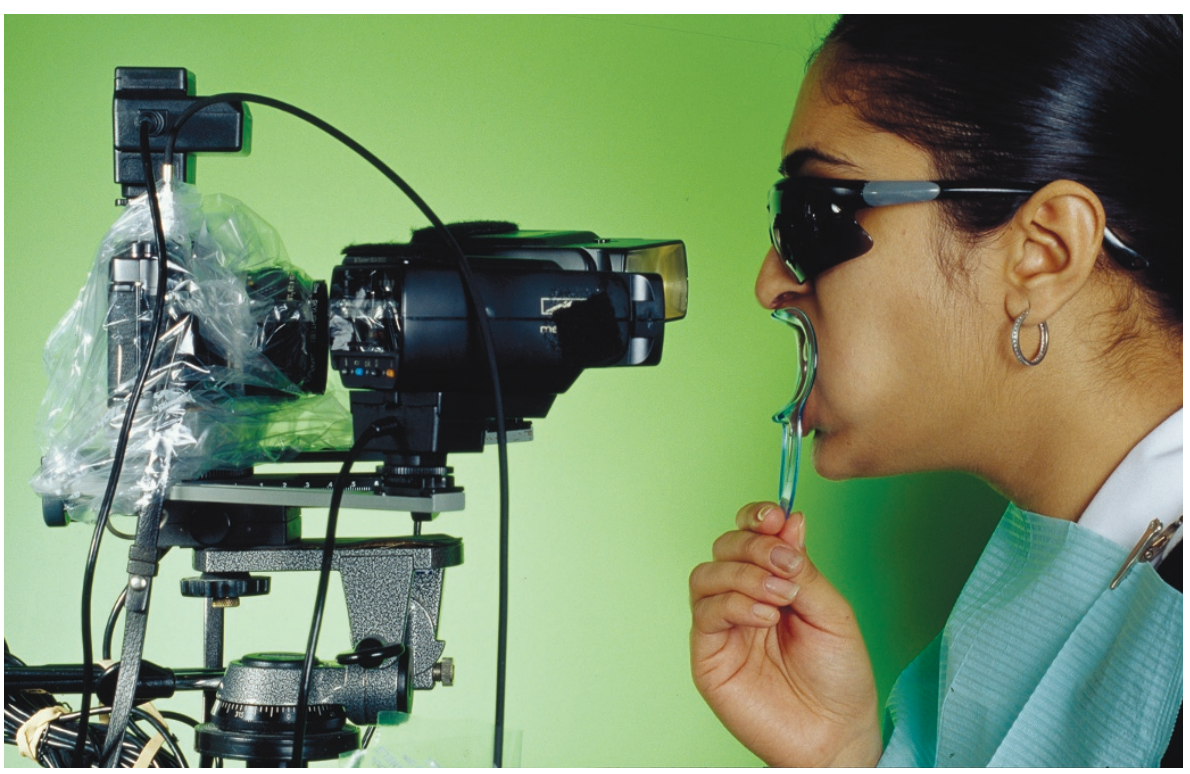

Fig. 1 Photographic equipment should be wrapped with disposable cellophane covers

\section{CROSS INFECTION CONTROL}

Unlike other forms of photography, dental photography requires strict adherence to cross-infection control measures. Routine cross-infection measures carried out for dental procedures are also applicable for dental photography. It is recommended that a specific zone be reserved for photographic equipment and accessories. All photographic equipment, including the camera, lens, tripod (if used) and cable releases should be draped with disposable cellophane covers, similar to that used for chair headrests (Fig. 1). Cheek retractors can either be autoclaved or cold sterilised, depending on the manufacturer's instructions. Intra-oral mirrors should be cleaned with cotton gauze soaked in a mild surface disinfectant, not surgical spirit, which causes smears or irreparable damage of the rhodium coating. Finally, all intra-oral photographic reflector and backdrop cards should be discarded after use.

\section{GENERAL GUIDELINES}

Most intra-oral images necessitate using cheek retractors and/or intra-oral photographic mirrors. The following general guidelines are applicable for all types of intra-oral photography. The first step is asking the patient to wear safety glasses. Unless stains or bio-film are being documented, the teeth should be flossed and polished with prophylaxis paste to remove food debris, plaque and stains which mask intrinsic tooth shade and texture. In order to facilitate placement of cheek retractors, petroleum jelly is copiously applied to the lips, taking care not to smear the teeth. This lubrication also prevents chapping 


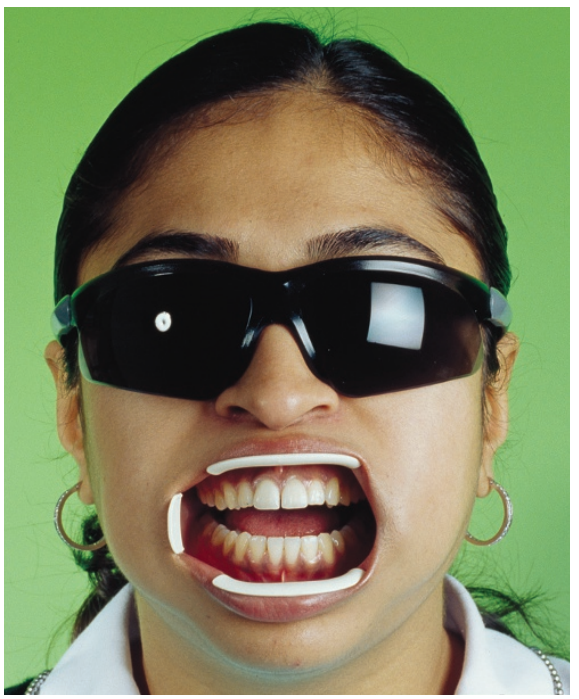

Fig. 2 Patient with bilateral cheek retractors for photographing the oral cavity

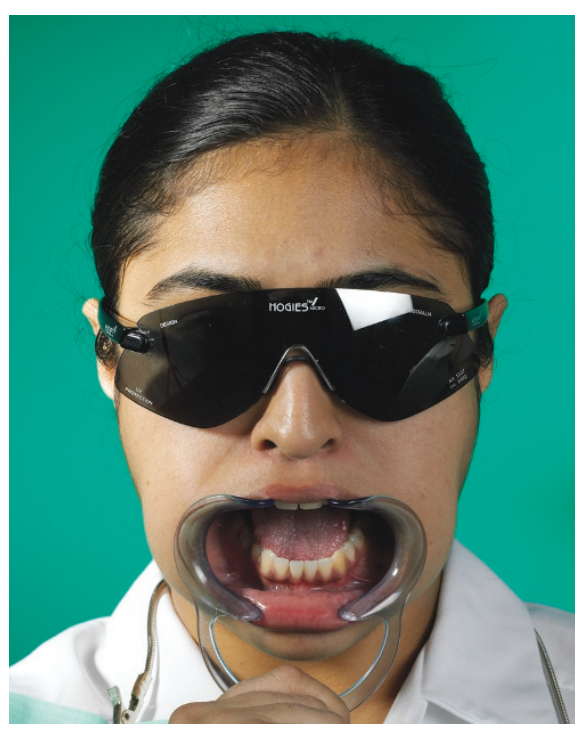

Fig. 3 A maxillary tooth will appear longer if the patient's chin is pointing downwards or the camera axis is superior to the maxillary arch, but this is a useful set-up for photographing mandibular anterior teeth

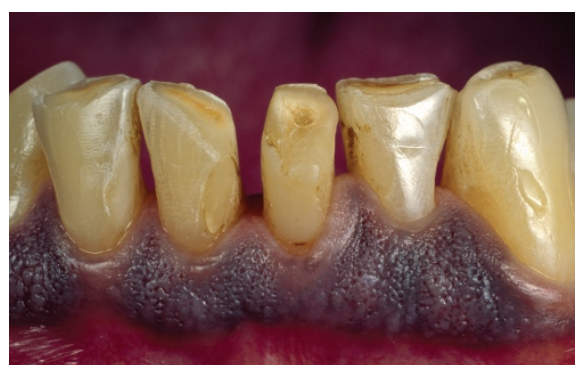

Fig. 4 Mandibular anterior teeth photographed using the set-up described in Figure 3

of the lips while they and the cheeks are displaced by the retractors.

The choice of cheek retractor is a personal preference, and also depends on the extent of mouth opening of the patient (Fig. 2). Many sizes and varieties are

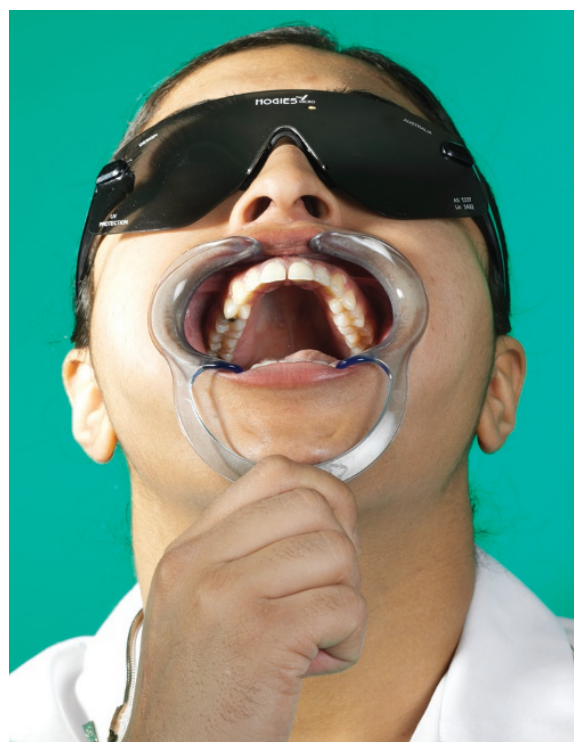

Fig. 5 A maxillary tooth will appear shorter if the patient's chin is pointing upwards or the camera axis is inferior to the maxillary arch. This is a useful set-up for illuminating and capturing crown margins

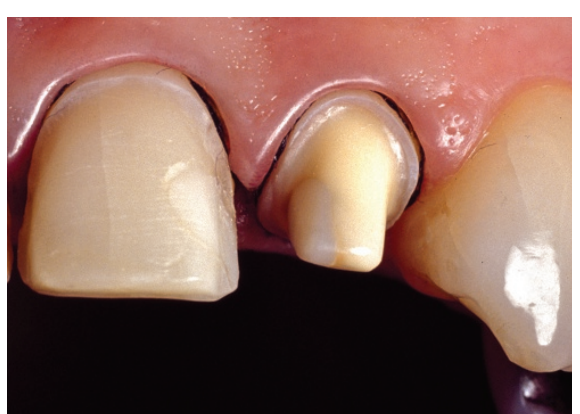

Fig. 6 Note the clearly visible crown margins of the left lateral incisor in this picture taken using the set-up described in Figure $\mathbf{5}$ compared to Figure 7, when the patient is looking directly into the camera (set-up described in Figure 8)

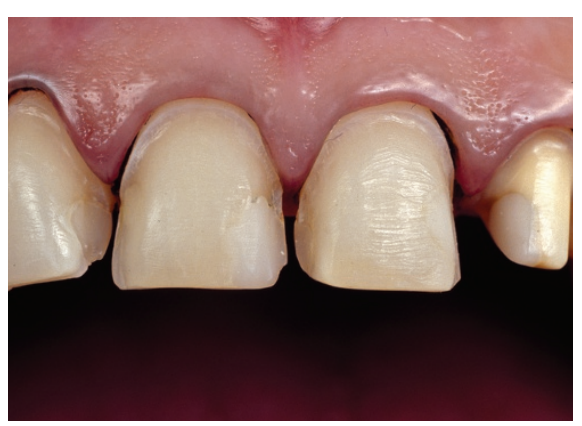

Fig. 7 When the patient is looking directly into the camera the crown margins of the left lateral incisor are not clearly discernible (compare with Figure 6)

available on the market and it is useful to have a selection to hand to cater for different eventualities. For full arch images the bilateral variety is preferred, while the unilateral retractor is more suitable for photographing individual quadrants.
Alternatively the dental nurse can stand behind the patient to hold two unilateral cheek retractors for full arch images, but this occupies the nurse who may be required for subsequent stages of the photographing procedure. After placing the cheek retractors, the patient is given tissues to wipe excess salivary flow. The teeth and gingivae are gently dried with a warm stream of air without desiccating the teeth. Other adjuncts to ensure a dry environment are saliva ejectors and cotton wool rolls, discretely placed so that they are not visible in the photograph.

As mentioned in Part $4,{ }^{1}$ it is highly advisable to mount the camera and flashes onto a tripod. A tripod not only serves as a solid platform, but also frees the operator's hand to concentrate on taking the photograph, reduces fatigue, and prevents potential accidents such as inadvertently bumping into surgery equipment. To ensure that a picture is correctly orientated, the camera axis and patient's head should be parallel in the horizontal, vertical and sagittal planes. Misalignment of the horizontal plane can convey incorrect assumptions, for example a slanted incisal plane, which is especially critical if anterior crowns are being planned. In the superiorinferior plane, incorrect alignment results in pseudo-shortening or lengthening of teeth in the inciso-gingival perspective, for example the maxillary teeth appear longer if the patient's chin is pointing downwards or the camera axis is superior to the maxillary arch. However, this is a useful set-up for photographing the mandibular anterior teeth (Figs 3-4). Conversely, shorter teeth are recorded if the chin is pointing upwards or the camera axis is inferior to the maxillary arch. This type of set-up is very useful for illuminating and capturing crown margins (Figs 5-7). While an incorrect horizontal plane is readily corrected in photo-editing software, an incorrect tooth perspective (superior-inferior plane) is difficult or almost impossible to rectify without introducing further distortions. This is particularly significant for magnification or detailed views.

Some clinicians prefer to delegate dental photography to another member of the dental team, for example a nurse, hygienist or dental technician. While this obviously saves time, especially in a busy practice, the resultant images may not yield 
the anticipated information. Ideally, it is the clinician's duty to take photographs since he or she knows what to concentrate on. Asking someone else to take stock, predefined views of each patient may not reveal clinically relevant information. For example, stock images may not record oral lesions of a patient who complains of symptoms of oral pathology, or document mamelons in a natural tooth in order that the ceramist can mimic this in a crown on an adjacent tooth. On the other hand, standardised views are indispensable for assessing occlusal relationships or tooth movement during orthodontic treatment. Furthermore, to achieve photographically acceptable results, training and experience in dental photography is a prerequisite. To summarise, each patient has different photographic requirements depending on the proposed treatment, and therefore the ideal person to make this judgement is the clinician, who is also probably more experienced in dental photography.

The following sections describe the setups necessary for photographing the different types of dental views.

\section{FULL-ARCH}

Two types of full arch images are required, frontal and occlusal.

\section{Full arch: frontal view (Figs 8-9)}

1. Ask the patient to bite in centric occlusion

2. Frame picture with as many teeth as possible in the composition, ideally including the second molars, while ensuring that cheek retractors and cotton wool rolls are not visible

3. Use the canines as the point of focus for ensuring maximum depth of field. The point of focus will depend on the shape of the upper and lower arches, and if all teeth are not in focus, change the point of focus either anterior or posterior to the canines

4. Take pictures in various mandibular positions as desired, including centric occlusion, protrusive and lateral excursions.

\section{Full arch: occlusal view} (Figs 10-13)

Occlusal and lateral views require using intra-oral photographic mirrors. Intra-orally,

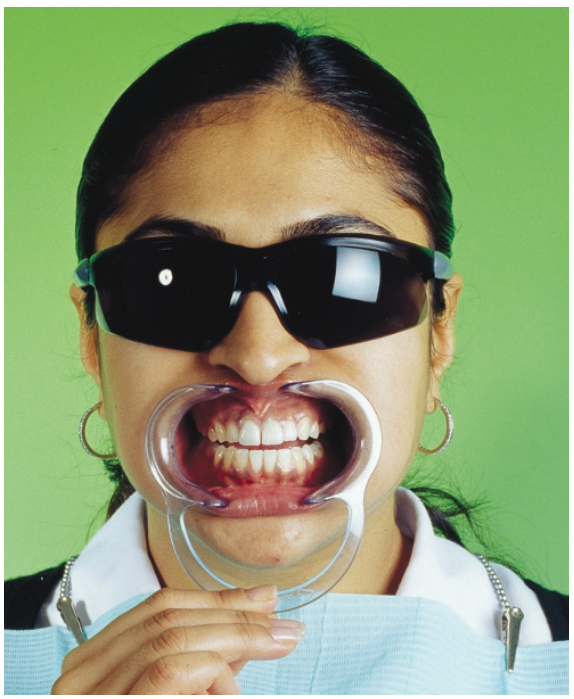

Fig. 8 Full arch frontal set-up

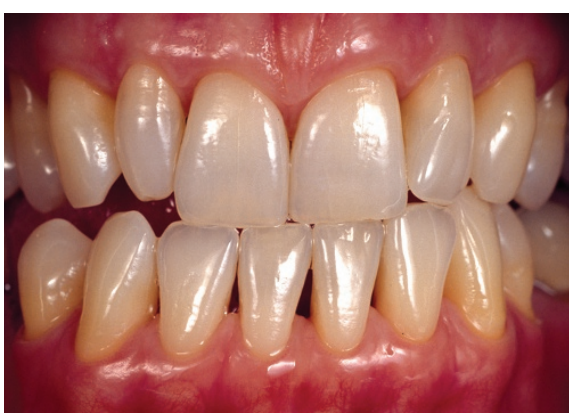

Fig. 9 Full arch frontal image

mirrors present a potential hazard and it is essential to avoid undue pressure on the soft tissues or hard tissues to prevent inadvertent breakage or shattering, with obvious consequences. The set-up is identical to that described previously for full arch pictures, with the following differences:

1. Select an appropriate sized occlusal mirror depending on the extent of mouth opening, and place gently in the mouth. For the maxillary arch, place mirror downwards, and for mandibular arch upwards, respectively

2. Request the patient to breathe through their nose if possible and ask the dental assistant to constantly blow warm dry air onto the mirror to prevent condensation

3. Focus onto the reflected image of the teeth on the mirror surface, framing as many teeth as possible. If mirror edges or actual teeth are in the frame these can be subsequently cropped during the image processing stage

4. Using mirrors means that light intensity is diminished because it has to travel a greater distance by being reflected off the mirror surface

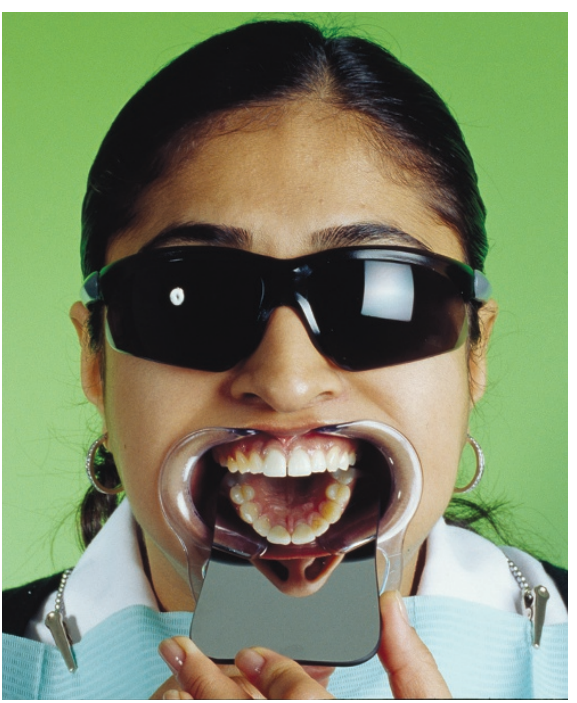

Fig. 10 Full maxillary arch occlusal set-up

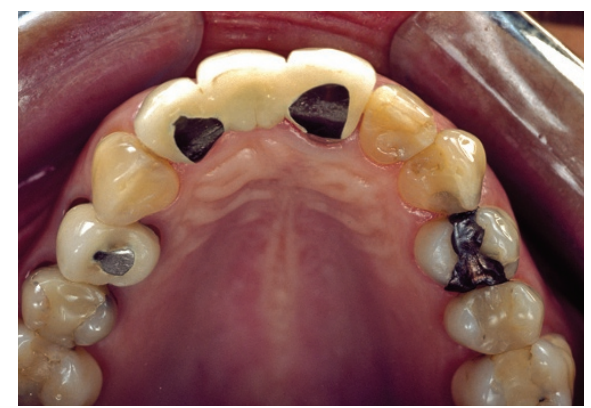

Fig. 11 Full maxillary arch occlusal image

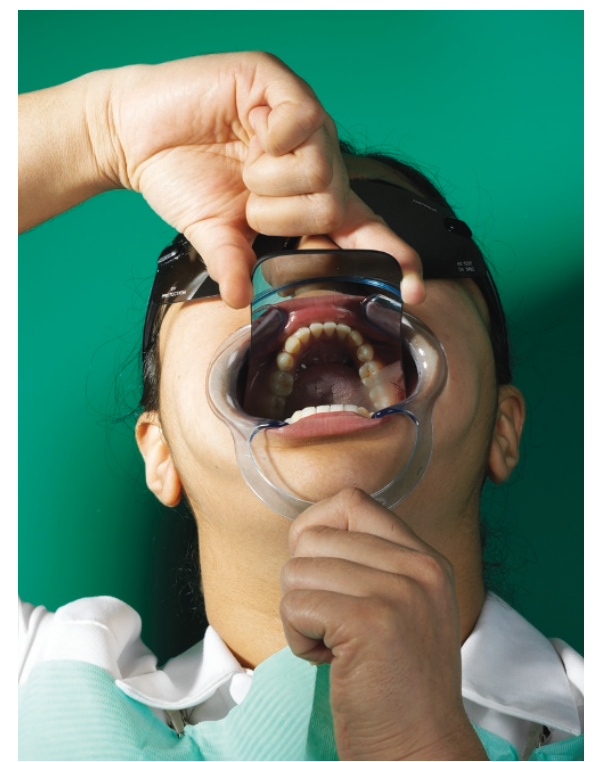

Fig. 12 Full mandibular arch occlusal set-up

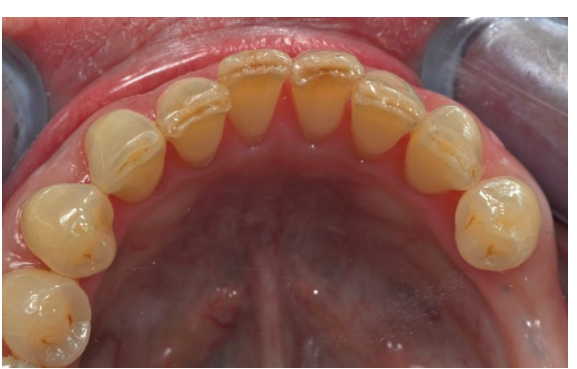

Fig. 13 Full mandibular arch occlusal image 


\section{PRACTICE}

before it reaches and illuminates the teeth. To compensate for the reduced illumination, either increase the light intensity or use a wider aperture, say f16, to ensure correct exposure.

\section{QUADRANT VIEWS: OCCLUSAL, LINGUAL AND BUCCAL}

The quadrant views record occlusal, lingual (or palatal) and buccal (lateral) surfaces of the teeth in an individual quadrant. The procedure is identical to taking full arch occlusal pictures, but the only difference is that narrower lateral mirrors are substituted for the wider full arch occlusal mirrors to concentrate on a specific quadrant or area. Also, unilateral cheek retractors are better suited to photographing quadrants since they are less cumbersome than the bilateral variety. As for all images using mirrors, moisture control is essential and a warm stream of air prevents condensation on the mirror surface (Figs 14-15).

Both lingual and buccal images also require narrower photographic mirrors. For buccal images, the patient is asked to close their mouth so that the buccinator muscles are relaxed, allowing room to place the mirror into the cheeks. Once again, unilateral cheek retractors may be less cumbersome than larger bilateral types. Images can be taken with the teeth fully occluded or slightly parted to concentrate on either the maxillary or mandibular teeth (Figs 16-17).

For palatal images of the maxillary teeth, the patient is asked to open the mouth and tilt the head backwards while the mirror is positioned to reflect the palatal aspects of the teeth. For improved access, a supine position is favoured for these types of images (Figs 18-19).

Lingual surfaces of mandibular teeth present two unique problems. Firstly, the sublingual salivary gland openings present a challenge for moisture control, and secondly, the proximity of the tongue causes difficulty for correct positioning of a mirror. In addition, excessive pressure may elicit a gagging reflex. Therefore for these types of views, time, practice and patience are necessary to achieve satisfactory results (Figs 20-21).

\section{MAGNIFICATION VIEWS}

If only particular parts of the oral mucosa or teeth are of interest, it is best to compose

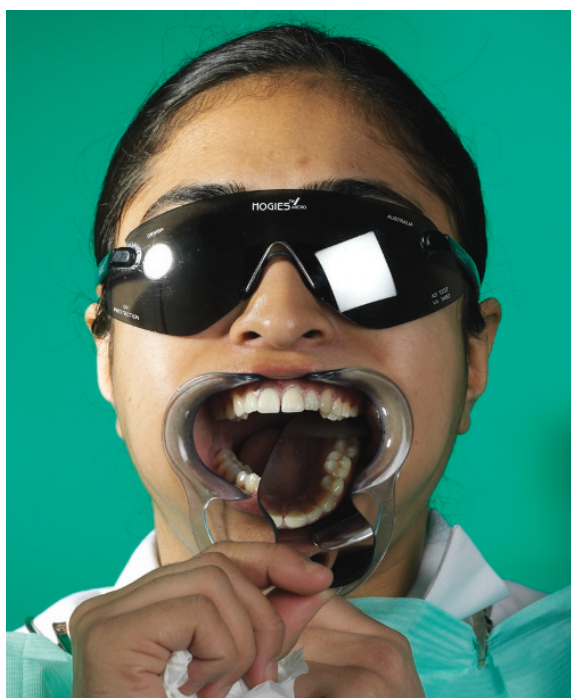

Fig. 14 Quadrant occlusal set-up

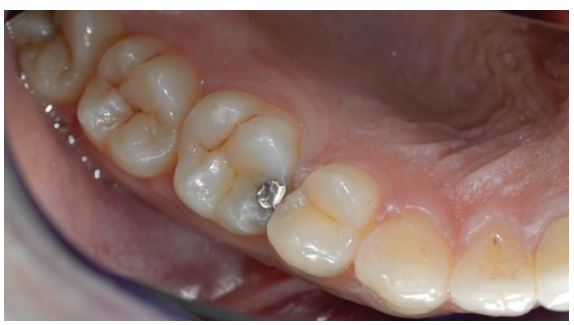

Fig. 15 Quadrant occlusal image

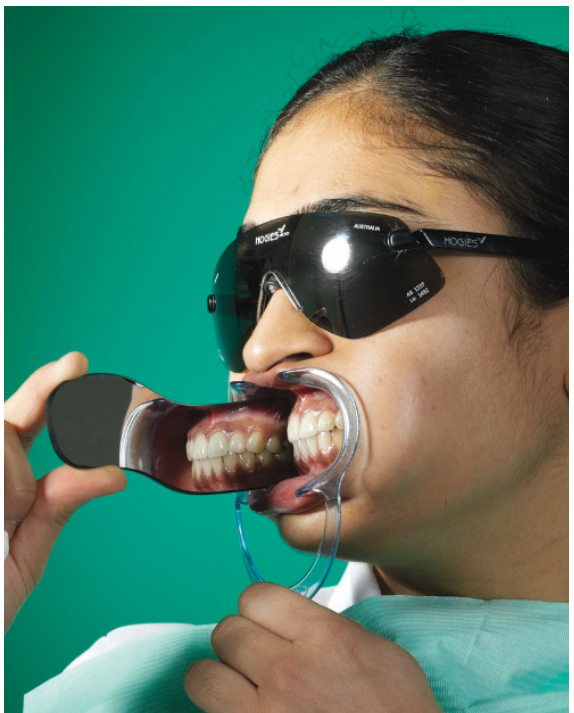

Fig. 16 Quadrant buccal set-up

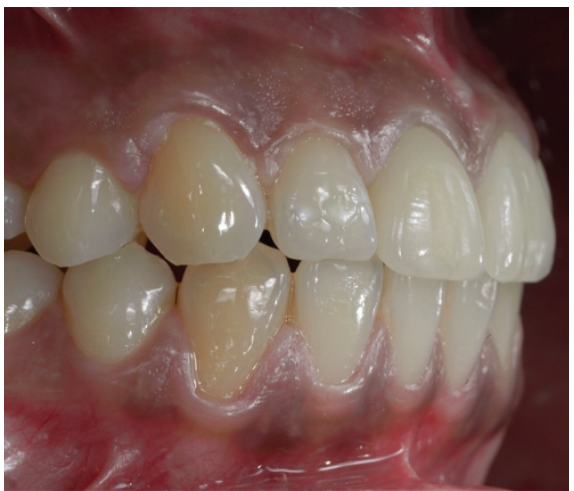

Fig. 17 Quadrant buccal image

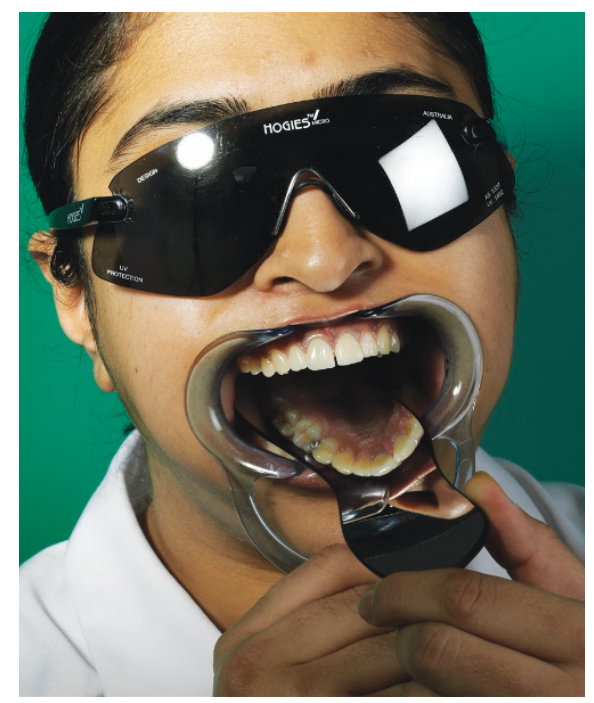

Fig. 18 Quadrant palatal set-up



Fig. 19 Quadrant palatal image

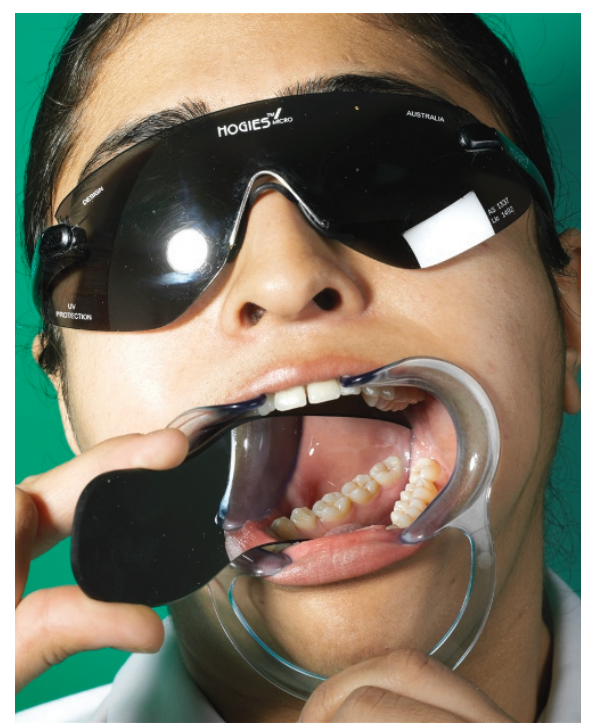

Fig. 20 Quadrant lingual set-up

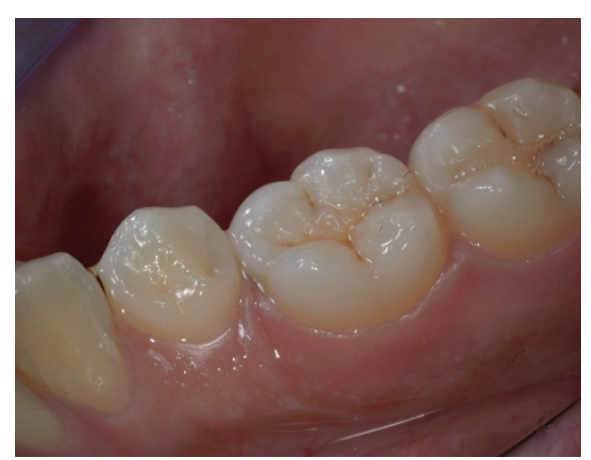

Fig. 21 Quadrant lingual image 
the picture concentrating on the desired areas. As a general rule, excess magnification is detrimental to image quality. This is because most macro lenses are incapable of resolving beyond a 1:1 magnification, and while it is possible to magnify objects to greater than life size using various attachments such as extension tubes and bellows, the result is a deterioration of image quality. If a magnification greater than $1: 1$ is required it is better to enlarge the image, again within limits, using photo-editing software. This is one of the reasons to start with a high quality image that is capable of enlargement without loss of detail.

The major factor to consider when taking magnification views is that the depth of field is substantially reduced, sometimes as small as $2 \mathrm{~mm}$. This means that fewer teeth or parts of teeth are sharply focused, not forgetting that the depth of field is in front as well as behind the point of focus. Therefore, to ensure maximum depth of field it is advantageous to focus on a midpoint. While framing of a picture is not critical since the image can be cropped afterwards, incorrect focusing is difficult to rectify in photo-editing software. A certain degree of sharpening can be applied, but if an image is captured out of focus it will remain and appear out of focus no matter the amount of manipulation. A tripod is invaluable for precise focusing and accurate framing. With a 1:1 magnification and assuming normal tooth alignment, the ideal point of focus is the distal aspect of the maxillary central incisors for maximum depth of field, which can also be verified by using the depth of field preview button. Finally, if no indication is given on the macro lens regarding the degree of magnification, an easy method for ensuring a 1:1 magnification is when four maxillary incisors occupy the entire viewfinder of a $35 \mathrm{~mm}$ DSLR camera (Figs 22-23).

\section{ORAL MUCOSA}

Photographing the oral mucosa and gingivae is similar to photographing teeth but requires minor modifications in technique. Firstly, disease is painful and therefore extra care is necessary to avoid trauma when placing retractors and mirrors if the tissues are inflamed. Mirrors are essential if pathological lesions are located in the deep recesses of the oral cavity. However, if trismus is present mirrors are not

recommended for safety reasons as well as those of limited access. For maximum comfort and depending on the site of the lesion, the patient can either be seated or placed in the supine position. If not contra-indicated, administration of topical or injectible local anaesthesia helps alleviate pain and expedite the photographic session. When composing the picture, ensure that a healthy area beyond or encircling the lesion is visible for comparison between healthy and diseased or pathologically altered tissue. Also, to assess the size of a lesion, placing a periodontal probe or millimetre scale adjacent to the pathology is helpful. The intensity of the photographic light needs to be increased to illuminate poorly lit posterior regions. Finally, colour is important when photographing soft tissues, as changes from the norm often indicate present or previous pathology. Therefore, calibrating with

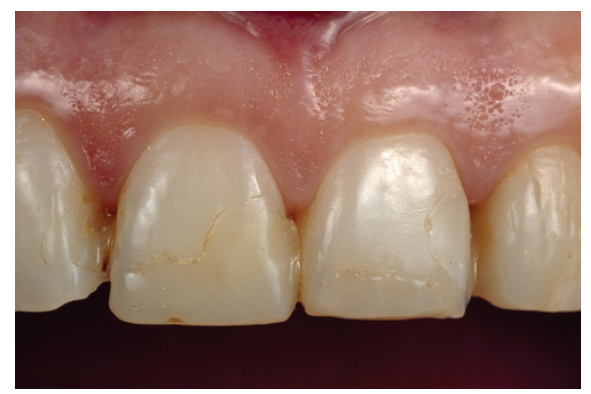

Fig. 22 1:1 magnification image

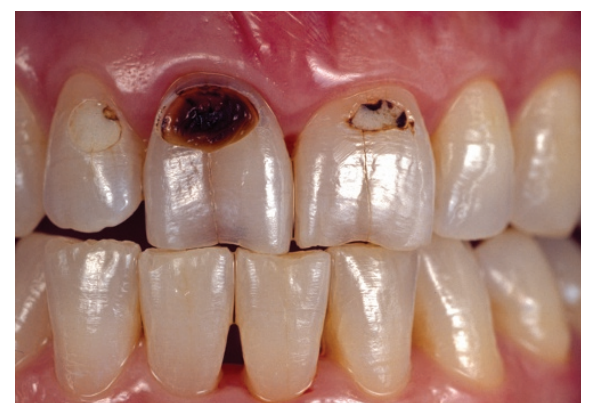

Fig. 23 1:1 magnification image



Fig. 24 Set-up to capture dentine strata or enamel cracks. A silver reflector is placed on the contra-lateral side of the flash to bounce light back onto the teeth

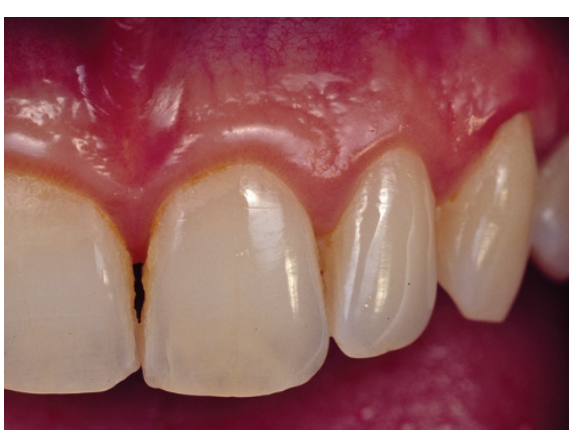

Fig. 25 Image taken with bilateral flashes showing the specular reflections that obscure dentine strata and enamel cracks



Fig. 26 The same image as Figure 25, but photographed with the set-up described in Figure 24, which reveals enamel cracks 
an $18 \%$ grey card is advisable for precise colour rendition.

\section{TEXTURE, DENTINE LAYER AND ENAMEL CRACKS}

Pictures that reveal texture, dentine strata, enamel cracks and perikymata are invaluable for a ceramist for visualising and subsequently mimicking these characterisations in artificial prostheses. The standard lighting for intra-oral images, ie two bilateral flashes mounted on either side of the camera, is the ideal configuration for capturing texture and lustre by specular reflection off the enamel surface. However, specular reflection masks the underlying dentine colour and characterisations as well as subtleties such as enamel cracks. To visualise the latter, a silver or white card is used to cover one of the bilateral lights, acting as a reflector to bounce muted light from the opposite flash onto the teeth without specular reflection. This set-up reveals the underlying dentine strata or intraenamel nuances (Figs 24-27). For this type of image, continuous light sources (LED or dental operatory lights) are advantageous because the reflector can be angled until the desired structures, for example dentine layer or perikymata, are visible in the viewfinder before taking the picture.

Failure to clearly visualise and capture cracks or other characterisations can result in artificial prostheses that do not mimic adjacent natural teeth. In Figure 28, the crown on the right central incisor is devoid of an enamel crack, which is clearly discernible on the natural left central incisor.

\section{TRANSLUCENCY - INCISAL AND INTERPROXIMAL}

Enamel translucency is usually located at the incisal edges, cusps and interproximal regions of a tooth. The ability to map the extent, degree and shape of translucencies and mamelons is invaluable for communication between the clinician and ceramist when fabricating artificial restorations. The technique is as follows. A black card, appropriately cut to size, is placed behind the teeth to obscure the oral cavity and a $1: 1$ magnification is chosen to concentrate on the desired teeth (Figs 29-30). Since black is an optical contrast to the white teeth, the camera's automatic metering may compensate by overexposing the

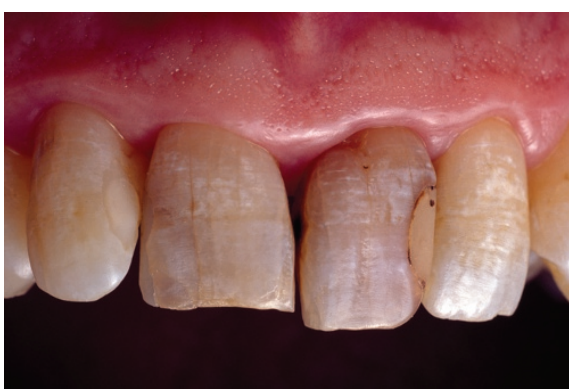

Fig. 27 Dentine strata and enamel cracks are clearly discernible using the set-up described in Figure 24



Fig. 28 The crown on the right central incisor is devoid of the fracture line that is clearly visible in the enamel of the natural left central incisor

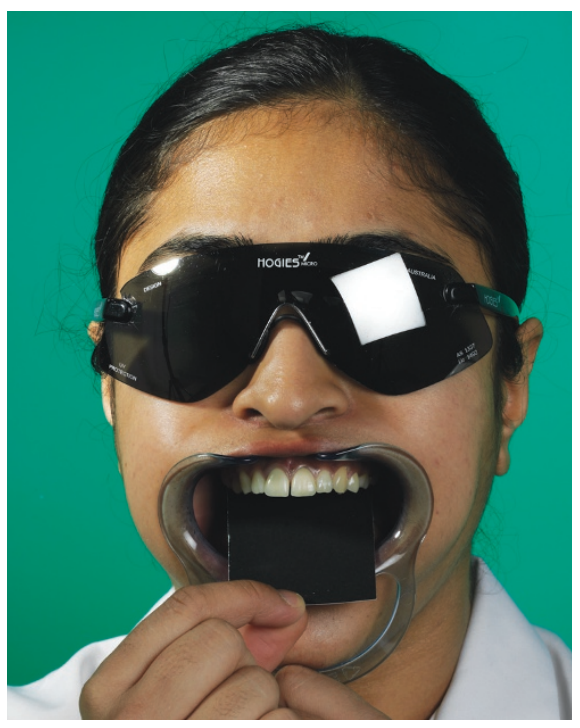

Fig. 29 A black card is placed behind the teeth to highlight enamel translucency

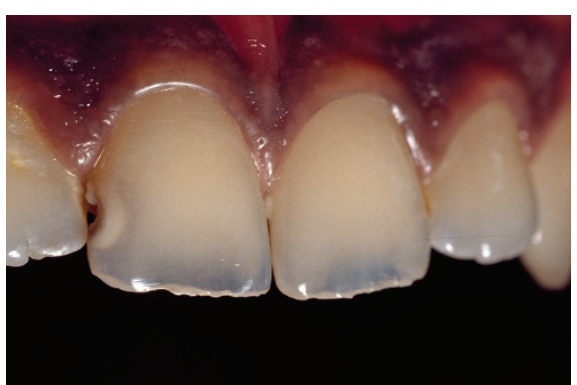

Fig. 30 Enamel translucency is clearly visible in this image taken with the set-up described in Figure 29

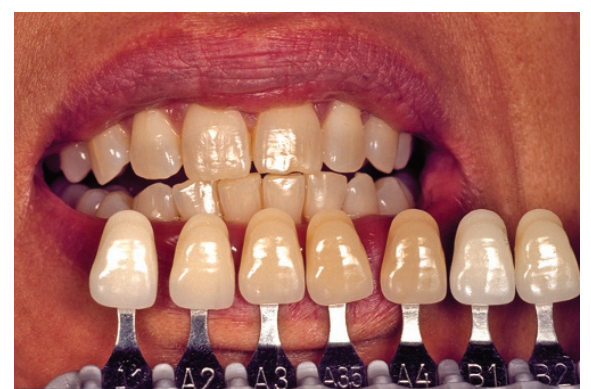

Fig. 31 A cursory shade analysis is carried out pre-operatively before bleaching

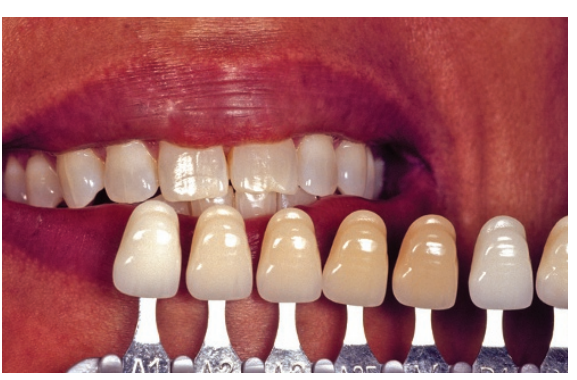

Fig. 32 The patient in Figure 28 two weeks after bleaching

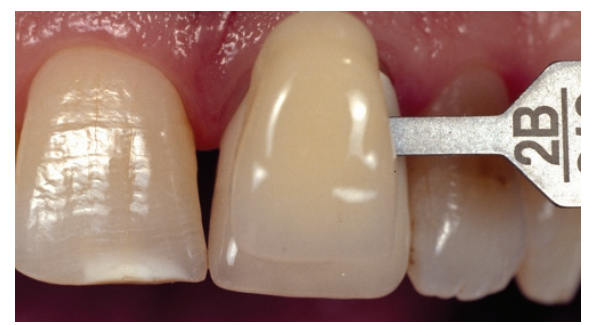

Fig. 33 A detailed shade analysis is performed pre-operatively with a single shade tab placed adjacent to a natural tooth

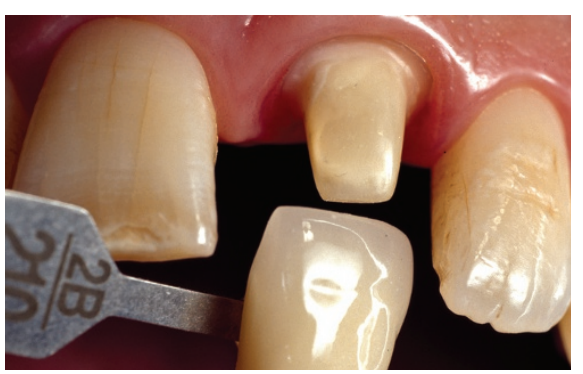

Fig. 34 A detailed shade analysis performed after tooth preparation with a single shade tab placed adjacent to the prepared tooth

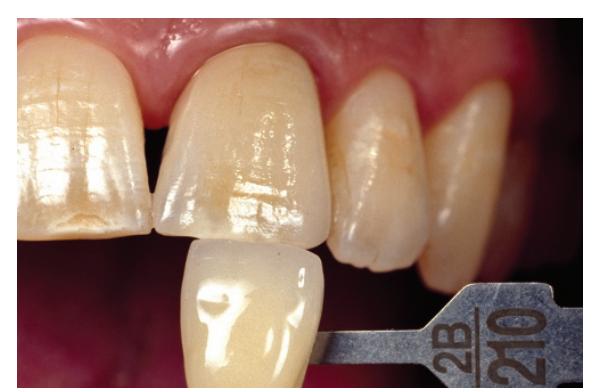

Fig. 35 A detailed shade analysis performed at the try-in stage using a single shade tab placed adjacent to the ceramic crown 
image by $1 / 2$ or $1 \mathrm{f}$-stop. This will diminish the visibility of translucency and it may be necessary to override the metering by underexposing to obtain correct exposure. As for recording texture and lustre, a continuous lighting source is helpful to visualise translucent areas by angling the light until the translucent areas or mamelon distribution are visible in the viewfinder.

\section{SHADE ANALYSIS}

Absolute tooth shade comparisons are impossible with photography alone and must be considered in combination with ocular or instrumental analysis. However, relative shade analysis is feasible with photography and is a useful guide for the dental ceramist for assessing the progress and extent of bleaching relative to a shade guide.

Photographs for shade are taken using a standard bilateral flash lighting set-up. As for the oral mucosa, calibration with a grey card is essential. The teeth should be moist and not unduly desiccated, especially if using a rubber dam, which causes inaccurate shade assessment. While moisture control is still necessary, saliva should be encouraged to flow over the teeth, simulating a natural oral environment. The patient is asked to hold the entire shade guide for a cursory analysis, or to hold individual tabs adjacent to the concerned teeth. Shade analysis with shade guides is useful for assessing the extent of bleaching (Figs 31-32). For a more precise shade comparison, a shade tab can be placed adjacent to a tooth that requires a crown. Ideally, shade analysis should be performed preoperatively, after tooth preparation and at the try-in stage, especially if the treatment objective is to whiten or brighten the shade of the definitive restorations (Figs 33-35).

If the shade of a natural tooth does not match those in a standard shade guide it may be necessary to fabricate a custom shade tab. As with conventional shade tabs, if the shade matching is proving

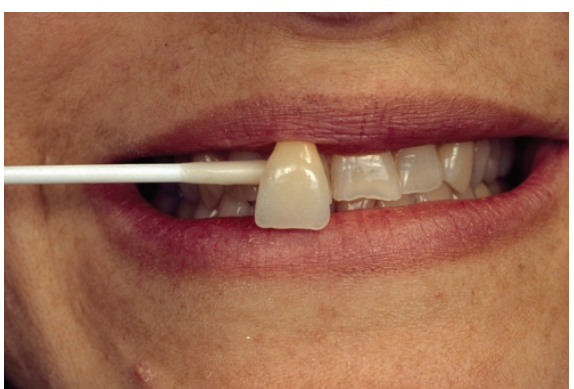

Fig. 36 A custom shade tab photographed with electronic flash with a colour temperature of 5,500 $\mathrm{K}$ appears to match the surrounding dentition

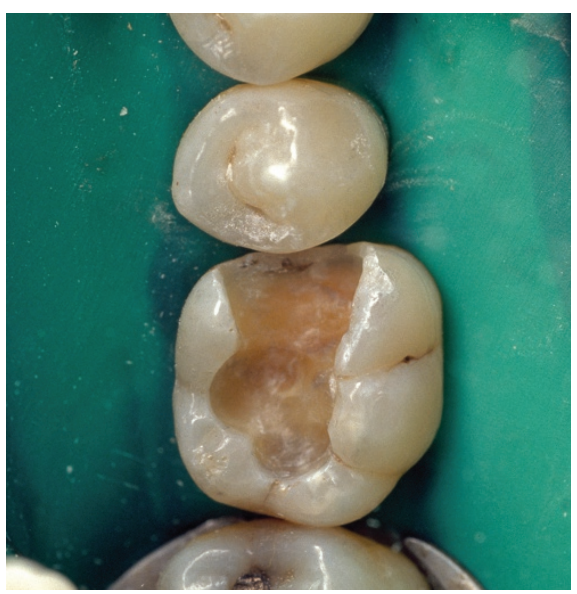

Fig. 38 Inlay preparation in mandibular right first molar

difficult photographs should be taken with illumination of different colour temperatures. This helps to avoid metamerism and facilities the matching procedure (Figs 36-37).

\section{POSTERIOR TEETH}

Photographing posterior teeth is challenging because of limited access and poor illumination, especially with restricted mouth opening or excess salivation. The set-up is identical to that for photographing quadrant occlusal images, described above. Therefore, a supine position is preferred, allowing better manipulation of intraoral mirrors and ease of access for saliva ejectors. For photographing a few teeth

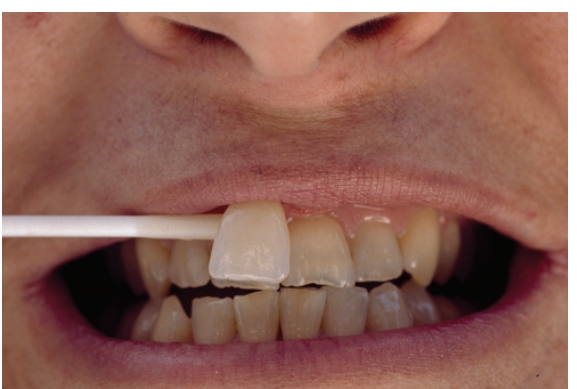

Fig. 37 The same shade tab as in Figure 36, photographed in natural daylight with a colour temperature of $6,500 \mathrm{~K}$, now has a higher value compared to the surrounding dentition

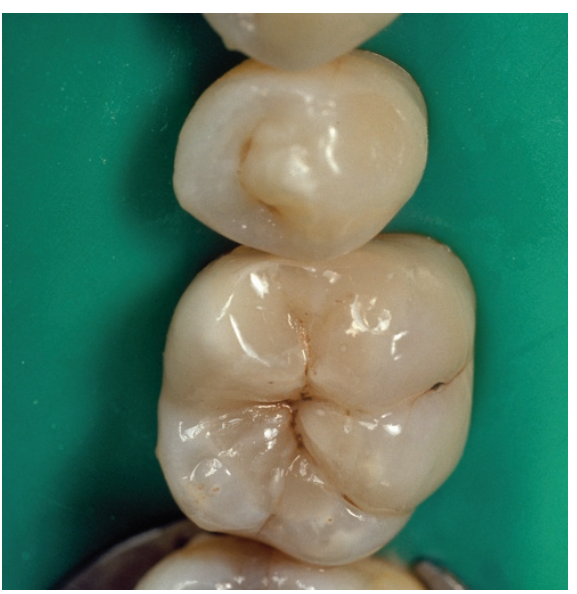

Fig. 39 Cemented inlay in mandibular right first molar

or a quadrant, unilateral cheek retractors and narrow occlusal mirrors are the ideal choice. A rubber dam is an excellent method of isolation, preventing condensation on the mirrors as well as safeguarding against ingestion or inhalation of dental instruments. These types of dental images are ideal for using ring flash illumination, which is intense, uniform and allows ease of manipulation in limited access areas. As previously mentioned, a slight overexposure may be necessary to compensate for light being reflected off the mirror surface to illuminate the teeth (Figs 38-39).

1. Ahmad I. Digital dental photography. Part 4: Choosing a camera and accessories. Br Dent J 2009; 206: 575-581. 